\title{
Análise faunística e flutuação populacional de cigarrinhas (Hemiptera, Cicadellidae) em pomar cítrico no município de Chapecó, Santa Catarina
}

\author{
Angela Camila Orbem Menegatti ${ }^{2}$ \\ Flávio Roberto Mello Garcia ${ }^{1,2 *}$ \\ Marcoandre Savaris ${ }^{2}$ \\ ${ }^{1}$ UNILASALLE, Setor de Pesquisa, Av. Victor Barreto, 2288, CEP 92010-000, Canoas, RS \\ ${ }^{2}$ UNOCHAPECÓ, Programa de Mestrado em Ciências Ambientais, Laboratório de Entomologia, Caixa Postal 747, Chapecó, SC \\ *Autor para correspondência \\ frmg@unilasalle.edu.br
}

Submetido em 13/03/2007

Aceito para publicação em 21/09/2007

\section{Resumo}

Visando caracterizar os ecossistemas de pomares cítricos da Região Oeste de Santa Catarina em relação às espécies de Cicadellidae, desenvolveu-se uma análise faunística em Chapecó. Para a caracterização do município foram medidos os índices de abundância, constância, dominância e freqüência das moscas das frutas. O levantamento foi feito utilizando-se armadilhas do tipo bandejas amarelas. Dilobopterus costalimai, Acrogonia citrina e Scopogonalia subolivacea foram as espécies mais abundantes, constantes, freqüentes e dominantes, podendo ser consideradas como predominantes. Dilobopterus costalimai apresentou dois picos populacionais, um em agosto e outro em dezembro e janeiro. Acrogonia citrina apresentou dois picos populacionais, um em agosto e outro em janeiro e fevereiro e Scopogonalia subolivacea apresentou um único pico populacional do mês de dezembro e janeiro.

Unitermos: cigarrinhas, dinâmica populacional, ecologia

\section{Abstract}

Faunistic analysis and population fluctuation of leafhoppers (Hemiptera: Cicadellidae) in citrus orchards at Chapecó, Santa Catarina, Brazil. In order to characterize the citrus orchard ecosystems of the western region of Santa Catarina state in relation to the Cicadellidae species, faunistic analysis was carried out in Chapecó from August to July 2005. To characterize the leafhopper population of this municipality, the abundance index, constancy, dominance and frequency were analyzed. The survey was made using yellow tray traps. Dilobopterus costalimai, Acrogonia citrina and Scopogonalia subolivacea were the most abundant, constant, frequent and dominant species, and were therefore considered as predominant. Dilobopterus costalimai had two population peaks, one in August and another in December-January. Acrogonia citrina had two population peaks, one in August and another in January-February. In contrast, S. subolivacea presented only a single population peak in period December-January.

Key words: leafhoppers, population dynamics, ecology 


\section{Introdução}

As cigarrinhas da família Cicadellidae, subfamília Cicadellinae podem atuar como vetores da Clorose Variegada do Citros (CVC) ou amarelinha, ao se alimentarem nos vasos do xilema de plantas cítricas (Paiva et al., 1996). A doença é comprovadamente transmitida por 11 espécies de cigarrinhas da família Cicadellidae: Acrogonia citrina Marucci \& Cavichioli, 2002, Acrogonia virescens Metcalf, 1949, Dilobopterus costalimai Young, 1977, Bucephalogonia xanthophis (Berg, 1879), Homalodisca ignorata Melichar, 1924, Ferrariana trivittata (Signoret, 1854), Macugonalia leucomelas (Walker, 1851), Oncometopia facialis (Signoret, 1854), Parathona gratiosa (Blanchard, 1840), Plesiommata corniculata Young, 1977 e Sonesimia grossa (Signoret, 1854) (Lopes, 1996; Gravena et al., 1997; Parra et al., 2003).

O agente casual da doença é a bactéria Xylella fastidiosa, que tem a característica de viver limitada ao xilema das plantas (Lee et al., 1993; Rossetti et al., 1990 e 1997). Esta bactéria é causadora de doenças em várias espécies de plantas, como videira, amendoeira, ameixeira, alfafa, pessegueiro, pereira, cerejeira, cafeeiro e outras (Purcell, 1989; Carlos et al., 1997), além disso, afeta todas as variedades comerciais de citros (Rossetti et al., 1997).

Os estudos sobre a flutuação populacional de cicadelídeos em pomares cítricos têm sido concentrados ao Estado de São Paulo (Paiva et al., 1996 e 2001; Garcia Jr. et al., 1997, Roberto e Yamamoto, 1998; Roberto et al., 2000; Yamamoto e Gravena, 2000; Yamamoto et al. 2002), havendo, portanto a necessidade de ampliar os estudos para outras áreas produtoras.

Entender como funciona a transmissão da CVC e os hábitos das cigarrinhas vetoras aumenta a possibilidade de se preverem suas características de agentes disseminadores de patógenos (Purcell, 1994). Tendo em vista as peculiaridades de cada região, os estudos com vetores em outras regiões talvez não sejam totalmente aplicáveis ao manejo na região sul, devido a possíveis diferenças na dinâmica populacional dos vetores. Portanto, este estudo teve o objetivo de identificar as cigarrinhas potenciais vetoras de CVC em pomar de citros no município de Chapecó, SC, através de análi- se faunística e flutuação populacional das espécies de cicadelídeos.

\section{Materiais e Métodos}

\section{Coleta}

Foram realizadas coletas semanais de agosto de 2004 a julho de 2005 em pomar de citros composto por: Citrus reticulata Blanco (cv. Tangerina poncã), C. deliciosa Temore (cv. Tangerina mexerica), C. sinensis Osbeck (cv. Valência, cv. Baía e cv. Tangor murcote). O pomar possui uma área de $300 \mathrm{mil} \mathrm{m}^{2}$, localizado as margens do Rio Uruguai $\left(22^{\circ} 15^{\prime} 17,5^{\prime}\right.$ 'S, $\left.57^{\circ} 40^{\prime} 53,5^{\prime \prime} \mathrm{W}\right)$ a altitude de $397 \mathrm{~m}$, sendo formado por plantas com idades de até 25 anos.

Foram utilizadas 30 armadilhas distribuídas aleatoriamente constituídas de bandejas de cor amarela (amarelo brilhante $n^{\circ} 3124$ ) instaladas sobre uma haste de madeira $1,50 \mathrm{~m}$ de altura do solo, localizado ao norte da planta, contendo dois litros de água, com três gotas de detergente neutro (Campos, 2002). Essas armadilhas possuem em um dos lados um orifício coberto com uma tela com $5 \mathrm{~cm}$, para evitar que com acúmulo de água das chuvas, a mesma transborde, perdendo o material entomológico. Semanalmente todos os insetos eram retirados das armadilhas e, após a remoção, era reposta a solução.

As cigarrinhas foram retiradas da bandeja filtrando-se a água com auxílio de um coador. Posteriormente foram colocadas em recipiente com álcool $70 \%$ e levadas para o Laboratório de Entomologia da UNOCHAPECÓ, para posterior identificação.

\section{Identificação e análises}

A identificação dos insetos foi realizada utilizando-se a chave de Marucci et al. (1999) e com auxílio do Prof. Dr. Rodney Ramiro Cavichioli do Departamento de Zoologia da Universidade Federal do Paraná.

A freqüência foi determinada através da porcentagem de indivíduos de cada espécie, em relação ao total de espécimes obtidos nas armadilhas em cada local, classificadas em pouco freqüentes, freqüentes e muito freqüentes (Thomazini e Thomazini, 2002). 
A medida faunística de constância foi determinada para cada espécie através da equação apresentada em Silveira Neto et al. (1976), sendo as espécies classificadas em constantes, acessórias e acidentais segundo Bodenheimer (1955, apud Silveira Neto et al., 1976).

O cálculo de abundância das populações e sua classificação em rara, dispersa, comum, abundante e muito abundante foram realizados de acordo com Garcia e Corseuil (1998).

As espécies foram classificadas em dominantes quando os valores de freqüência foram superiores ao limite calculado pela equação proposta por Sakagami e Laroca (1971, apud Silva, 1993).

\section{Resultados e Discussão}

Foram capturadas 4.500 cigarrinhas de 18 espécies: Acrogonia citrina, A. sagittaria (Walker,1858), Aulacizes obsoleta Melichar, 1926, Borogonalia impressifrons (Signoret, 1854), Dilobopterus costalimai, Diedrocephala variegata (Fabricius, 1775), Homalodisca ignorata, Macugonalia cavifrons (Stal, 1862), M. leucomelas, Molomea consolida Schröder, 1959, M. lineiceps Young, 1968, Oncometopia facialis, Parathona gratiosa, Scopogonalia penicula Young, 1977, S. subolivacea (Stal, 1862), Sibovea sogata (Signoret, 1854), Sonesimia grossa e Teletusa limpida (Signoret, 1855). Dessas espécies, apenas sete são comprovadamente transmissoras da $X$. fastidiosa: A. citrina, $D$. costalimai, $H$. ignorata, M. leucomelas, $O$. facialis. $P$. gratiosa e $S$. grossa.

As espécies mais freqüentes foram $D$. costalimai (37,8\%), A. citrina $(30,6 \%)$ e S. subolivacea $(9,3 \%)$ (Tabela1). Yamamoto et al. (2002) constataram que Acrogonia sp foi a segunda espécie mais freqüente em viveiro de citros localizado em Mogi-Guaçu, SP, representando $26,7 \%$ do total de cigarrinhas capturadas. Santos et al. (2005) observaram que A. citrina e D. costalimai foram as espécies mais freqüentes em Viçosa, MG, representando respectivamente $7,4 \%$ e $11,3 \%$ do total capturado em armadilhas adesivas. Yamamoto et al. (2001) também encontrou $A$. citrina e D. costalimai como principais cicadelídeos capturados em pomar localizado em Nova Europa, SP.

As espécies de ocorrência constantes foram $A$. citrina, D. costalimai, M. cavifrons, M. consolida, $O$. facialis, $P$. gratiosa, e $S$. subolivacea. As espécies $A$. sagittaria, $H$. ignorata, $M$. leneiceps, $S$. penicula e $S$. sogata foram classificadas como acessórias. Já, as espécies $A$. obsoleta, B. impressifrons, D. variegata, $M$. leucomelas, $S$. grossa e T. limpida foram acidentais (Tabela 1). Campos (2002) verificou que Acrogonia sp, $D$. costalimai e $O$. facialis foram constantes em pomar de citros em Cunha-Porã, SC. Yamamoto et al. (2002) constataram Acrogonia sp foi constante e D. costalimai foi acidental em São Paulo. Santos et al. (2005) detectaram que as espécies $A$. citrina, D. costalimai e $O$. facialis foram constantes em Minas Gerais.

As espécies $A$. citrina, D. costalimai e $S$. subolivacea podem ser consideradas predominantes, uma vez que foram classificadas como constantes, dominantes, muito freqüentes e muito abundantes, além disso, $A$. citrina e $D$. costalimai são compravadamente vetores de CVC, sendo assim medidas de controle devem priorizar estas duas espécies.

Houve predominância de $D$. costalimai, sendo esta a mais numerosa, seguida por A. citrina e $S$. scopogonalia. Dilobopterus costalimai é mais comum em pomares em produção. As espécies Acrogonia sp, $D$. costalimai e $O$. facialis foram de ocorrência constante nas regiões Norte, Nordeste e Centro do Estado de São Paulo em pomares de laranjeiras em produção (Roberto e Yamamoto, 1998; Yamamoto et al., 2002).

As espécies de cicadelídeos foram capturadas no pomar de citros, durante todos os meses de coleta. Ocorreram dois picos populacionais, um ano mês de agosto, e o outro no mês de dezembro. No mês de agosto foram coletadas 757 cigarrinhas, correspondendo ao mês de maior incidência (Figura 1). A maior ocorrência de cigarrinhas para o mês de agosto provavelmente foi devido a condições climáticas favoráveis para os insetos. Segundo Paiva et al. (2001) a população de cigarrinhas tende a aumentar a partir do início das chuvas, 
verão e outono, e tende a diminuir no inverno e início da primavera. Em anos de estiagem prolongada a população de insetos apresenta picos mais tardios (Roberto e Yamamoto, 1998, Maruyama et al., 2002). Neste levantamento constatou-se que no verão e outono houve crescimento populacional, posteriormente, uma queda populacional, sendo que no inverno de 2005 e início de primavera de 2004 a população diminuiu, a única exceção foi no inverno de 2004, sendo que no mês de agosto ocorreu um aumento populacional.

Dilobopterus costalimai apresentou dois picos populacionais, um em agosto e outro em dezembro e janeiro resultados semelhantes foram obtidos por Campos (2002) no município de Cunha Porã, SC. Acrogonia citrina apresentou dois picos populacionais, um em agosto e outro em janeiro e fevereiro. Scopogonalia subolivacea apresentou um único pico populacional do mês de dezembro e janeiro, a partir do final do outono e inverno houve uma queda drástica na sua população (Figura 1).
Milanez et al. (2005) relatam que as áreas recomendadas para o plantio de citros no estado de SC, coincidem com as áreas mais favoráveis para o desenvolvimento populacional de cigarrinhas. A região Oeste é onde está concentrada a produção de citros e também a maior disseminação da $\mathrm{CVC}$, verificou que o número estimado de gerações de $D$. costalimai, $O$. facialis e $A$. citrina para esta região são altos, principalmente nas regiões próximas ao Vale do Rio Uruguai, onde as altitudes são menores e as temperaturas médias mensais são mais elevadas.

Os resultados evidenciam que em pomar em produção no oeste de Santa Catarina, predominam as cigarrinhas $D$. costalimai e A. citrina, sendo estas espécies comprovadamente transmissoras de $X$. fastidiosa, oferecendo grande risco de infecção para a citricultura catarinense.

TABELA 1: Análise faunística de cigarrinhas (Hemiptera: Cicadellidae) coletadas através de bandejas amarelas em pomar de citros de dezembro de 2004 a março de 2005, Chapecó-SC.

\begin{tabular}{|c|c|c|c|c|}
\hline Espécie & Abundância & Constância & Dominância & Freqüência \\
\hline D. costalimai & Muito abundante & Constante & Dominante & Muito freqüente \\
\hline A. citrina & Muito abundante & Constante & Dominante & Muito freqüente \\
\hline S. subolivacea & Muito abundante & Constante & Dominante & Muito freqüente \\
\hline M. consolida & Comum & Constante & Não dominante & Freqüente \\
\hline P. gratiosa & Dispersa & Constante & Não dominante & Muito freqüente \\
\hline M. cavifrons & Dispersa & Constante & Não dominante & Pouco freqüente \\
\hline O. facialis & Rara & Constante & Não dominante & Pouco freqüente \\
\hline M. lineiceps & Rara & Acessória & Não dominante & Pouco freqüente \\
\hline A. sagittaria & Rara & Acessória & Não dominante & Pouco freqüente \\
\hline S. penicula & Rara & Acessória & Não dominante & Pouco freqüente \\
\hline S. sogata & Rara & Acessória & Não dominante & Pouco freqüente \\
\hline H. ignorata & Rara & Acessória & Não dominante & Pouco freqüente \\
\hline M. leucomelas & Rara & Acidental & Não dominante & Pouco freqüente \\
\hline A. obsoleta & Rara & Acidental & Não dominante & Pouco freqüente \\
\hline D. variegata & Rara & Acidental & Não dominante & Pouco freqüente \\
\hline S. grossa & Rara & Acidental & Não dominante & Pouco freqüente \\
\hline B. impressifrons & Rara & Acidental & Não dominante & Pouco freqüente \\
\hline T. limpida & Rara & Acidental & Não dominante & Pouco freqüente \\
\hline
\end{tabular}


- Dilobopterus costalimai - * - Acrogonia citrina $\longrightarrow-$ Scopogonalia subolivacea

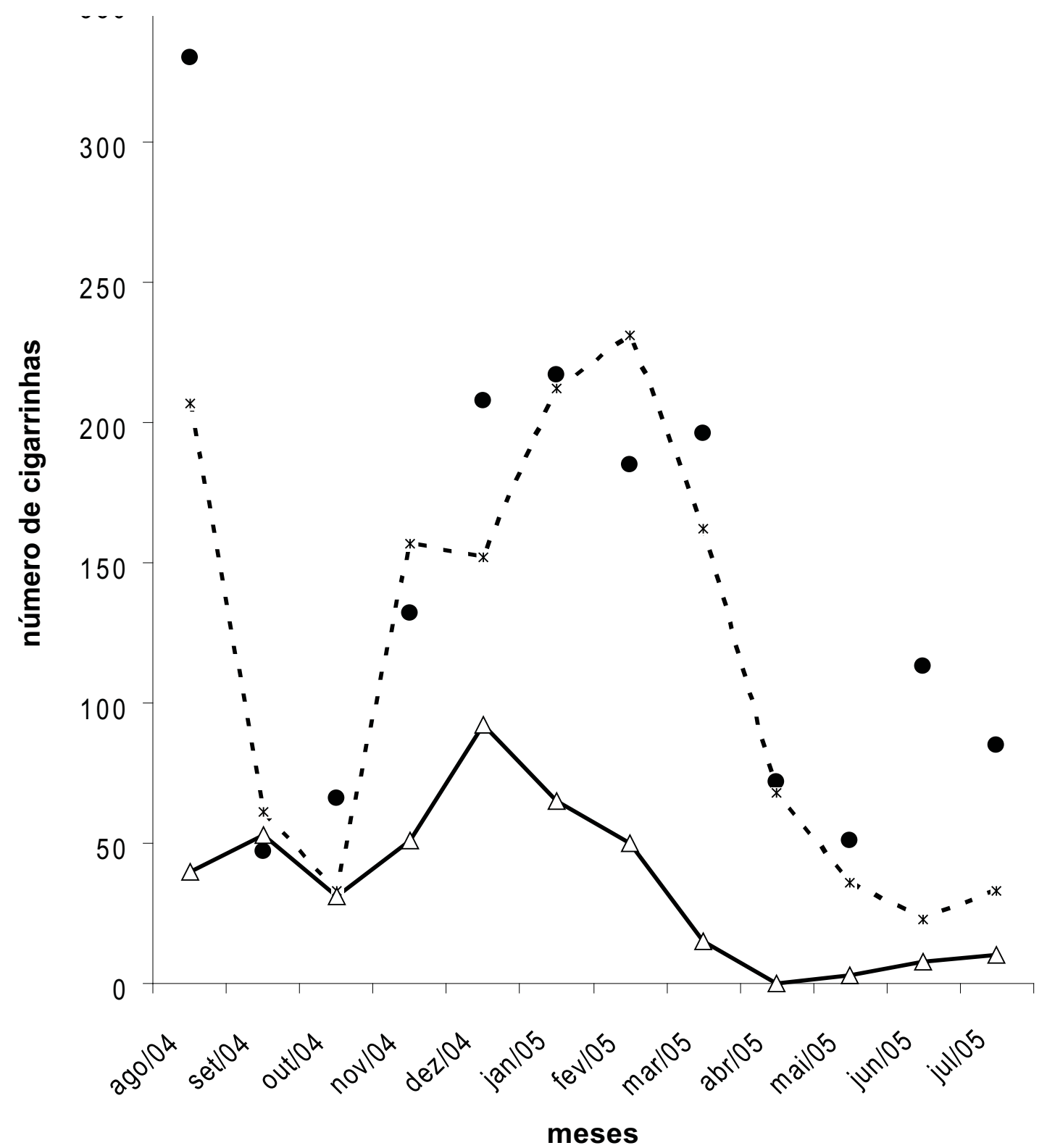

FIGURA 1: Flutuação populacional de cigarrinhas (Cicadellidae) coletadas com armadilhas de bandeja amarela em pomar de citros em Chapecó - SC, no período de agosto de 2004 a julho de 2005.

\section{Agradecimentos}

Ao Fundo de Apoio a Pesquisa (FAPE) da UNOCHAPECÓ pelo fomento da pesquisa. Ao Dr. Rodney Ramiro Cavichioli da UFPR pela confirmação e identificação de algumas cigarrinhas.

\section{Referências}

Campos, J. V. 2002. Dinâmica populacional de cigarrinhas (Hemiptera, Cicadellidae) vetoras clorose variegada no município de Cunha Porá, Santa Catarina. Trabalho de Conclusão de Curso, Universidade Comunitária Regional de Chapecó, Brasil, 60pp. 
Carlos, E. F.; Rodrigues Neto, J.; Beretta, M. J. G. 1997. A bactéria Xylella fastidiosa. In: Donadio, L. C. \& Moreira C. S. (eds). Clorose Variegada dos Citros. Estação Experimental de Citricultura, Bebedouro, Brasil, p.22-36.

Garcia, F. R. M.; Corseuil, E. 1998. Análise faunística de moscasdas-frutas (Diptera, Tephritidae) em pomares de pessegueiro em Porto Alegre, Rio Grande do Sul. Revista Brasileira de Zoologia, 15 (4): 1111-1117.

Garcia Jr., A.; Lopes, J. R. S.; Beretta, M. J. G. 1997. Population survey of leafhopper vectors of Xylella fastidiosa in citrus nurseries, in Brazil. Fruits, 52 (6): 371-374.

Gravena, S.; Lopes, J. R. S.; Paiva, P. E. B.; Yamamoto, P. T.; Roberto, E. R. 1997. Os vetores da Xylella fastidiosa. In: Donadio, L. C. \& Moreira C. S. (eds). Clorose Variegada dos Citros. Estação Experimental de Citricultura, Bebedouro, Brasil, p.37-53.

Lee, R. F.; Baretta, M. J. G.; Hartung, J. H.; Hooker, M. E.; Derrick, K. S. 1993. Xylella fastidiosa: Agente causal da Clorose Variegada dos citros. Laranja, 14 (1): 157-166.

Lopes, J. E. S. 1996. Mecanismos de transmissão de Xylella fastidiosa por cigarrinhas. Laranja, 17 (1): 79-92.

Marucci, R. R.; Cavichioli, R. R.; Zuchi, R. A. 1999. Chave para as espécies de cigarrinhas (Hem., Cicadellidae, Cicadellinae) vetoras da clorose variegada dos citros (CVC). Anais da Sociedade Entomológica do Brasil, 28 (3): 439-446.

Maruyama, W. I.; Barbosa, J. C.; Fernandes, M. G.; Yamamoto, P. T. 2002. Distribuição espacial de Dilobopterus costalimai Young (Hemiptera: Cicadellidae) em citros na região de Taquaritinga, SP. Neotropical Entomology, 31 (1): 35-40.

Milanez, J. M.; Pandolfo, C.; Hammes, L. A.; Parra, J. R. P. 2005. Zoneamento ecológico de Dilobopterus costalimai Young, Oncometopia facialis e Acrogonia citrina Marucci \& Cavichioli (Hemiptera: Cicadellidae) para Santa Catarina. Neotropical Entomology, 34 (2): 297-302.

Paiva, P. E. B.; Benvenga, S. R.; Gravena, S. 2001. Aspectos biológicos das cigarrinhas Acrogonia gracialis (Osborn), Dilobopterus costalimai Young e Oncometopia facialis (Signoret) (Hemiptera: Cicadellidae) em Citrus sinensis L. Osbeck. Neotropical Entomology, 30 (1): 25-28.

Paiva, P. E. B.; Silva, J. L. da; Gravena, S.; Yamamoto, P. T. 1996. Cigarrinhas de xilema em pomares de laranja no Estado de São Paulo. Laranja, 17 (1): 41-54.

Parra, J. R. P.; Oliveira, H. N.; Pinto A. de S. 2003. Pragas e insetos benéficos com Citros. FEALQ, Piracicaba, Brasil, 73pp.

Purcell, A. H. 1989. Homopteram transmission of xylem- limited bacteria. In: Harris, K. F. (ed.). Advances in vector research. Springer, New York, USA, p.243-266.
Purcell, A. H. 1994. Cigarrinhas na cultura de citros. In: Donadio, L. C. \& Gravena, S. (eds). Manejo integrado de pragas dos citros. Fundação Cargill, Campinas, Brasil, p.195-209.

Roberto, S. R.; Yamamoto, P. T. 1998. Flutuação populacional e controle químico de cigarrinhas em citros. Laranja, 19 (2): 269284.

Roberto, S. R.; Pria Júnior, W. D.; Yamamoto, P. T.; Fellipe, M. R.; Freitas, E. P. de. 2000. Espécies e flutuação populacional de cigarrinhas de citros em Gavião Peixoto (SP). Laranja, 21 (1): 65-79.

Rosseti,V.; Garnier, M.; Beretta, M. J. G.; Teixeira, A. R. R.; Quaggio, J. A.; Bataglia, O. C.; Gomes, M. P.; De Negri, J. D. 1990. Resultados preliminares de estudos sobre uma nova anormalidade dos citros observada nos Estados de São Paulo e Minas Gerais. Summa Phytopathologica, 16 (1): 13.

Rossetti, V.; Gonzales, M. A.; Donadio, L. C. 1997. Histórico. In: Donadio, L. C. \& Moreira, C. S. (eds). Clorose Variegada dos citros. Estação Experimental de Citrucultura, Bebedouro, Brasil, p.1-21.

Santos, D. dos; Siqueira, D. L. de; Picanço, M. C. 2005. Flutuação populacional de espécies de cigarrinhas transmissoras da clorose dos citros (CVC) em Viçosa, MG. Revista Brasileira de Fruticultura, 27 (2): 211-214.

Silva, N. M. da. 1993. Levantamento e análise faunística de moscas-das-frutas (Diptera: Tephritidae) em quatro locais do Estado do Amazonas. Tese de Doutorado, Universidade de São Paulo, Brasil, 152pp.

Silveira Neto, S.; Nakano, O.; Barbin, D.; Villa Nova, N. A. 1976. Manual de ecologia dos insetos. Agronômica Ceres, Piracicaba, Brasil, 419pp.

Thomazini, M. J.; Thomazini, A. P. de B. W. 2002. Diversidade de abelhas (Hymenoptera: Apoidea) em inflorescências de Piper hispidinervum (C.D.C.). Neotropical Entomology, 31 (1): 27-34.

Yamamoto, P. T.; Gravena, S. 2000. Espécies e abundância de cigarrinhas e psilídeos (Homoptera) em pomares cítricos. Anais da Sociedade Entomológica do Brasil, 29 (1): 169-176.

Yamamoto, P. T.; Pria Júnior, W. D.; Roberto, S. R.; Felippe, M. R.; Freitas, E. P. de. 2001. Flutuação populacional de cigarrinhas (Hemiptera: Cicadellidae) em pomar cítrico em formação. Neotropical Entomology, 30 (1): 175-177.

Yamamoto, P. T.; Roberto, S. R.; Pria Júnior, W. D.; Felippe, M. R.; Freitas, E. P. de. 2002. Espécies e flutuação populacional de cigarrinhas em viveiro de citros, no município de Mogi-Guaçu-SP. Revista Brasileira de Fruticultura, 24 (2): 389-394. 